\title{
Tactile Acuity is Enhanced in Blindness
}

\author{
Daniel Goldreich and Ingrid M. Kanics \\ Department of Occupational Therapy, Rangos School of Health Sciences, Duquesne University, Pittsburgh, Pennsylvania 15282
}

Functional imaging studies in blind subjects have shown tactile activation of cortical areas that normally subserve vision, but whether blind people have enhanced tactile acuity has long been controversial. We compared the passive tactile acuity of blind and sighted subjects on a fully automated grating orientation task and used multivariate Bayesian data analysis to determine predictors of acuity. Acuity was significantly superior in blind subjects, independently of the degree of childhood vision, light perception level, or Braille reading. Acuity was strongly dependent on the force of contact between the stimulus surface and the skin, declined with subject age, and was better in women than in men. Despite large intragroup variability, the difference between blind and sighted subjects was highly significant: the average blind subject had the acuity of an average sighted subject of the same gender but 23 years younger. The results suggest that crossmodal plasticity may underlie tactile acuity enhancement in blindness.

Key words: tactile acuity; crossmodal plasticity; blind; Braille; grating orientation; somatosensory psychophysics; sensory compensation

\section{Introduction}

Does blindness result in enhanced acuity in the remaining senses? Occipital cortical areas that normally subserve vision are activated in congenitally blind subjects performing auditory (Weeks et al., 2000) or tactile (Sadato et al., 1996, 1998, 2002; Cohen et al., 1999; Melzer et al., 2001) spatial discrimination tasks (crossmodal plasticity). In addition, within the primary somatosensory cortex of Braille readers, the representation of the reading finger is enlarged (unimodal plasticity) (Pascual-Leone and Torres, 1993). Nevertheless, the link, if any, between these different types of cerebrocortical plasticity and the perceptual acuity of blind persons remains unclear.

Auditory spatial localization is reportedly enhanced in blindness (Lessard et al., 1998; Röder et al., 1999), but whether blind people have enhanced tactile acuity has long been controversial (Axelrod, 1959; Warren, 1978; Hollins, 1989; Pascual-Leone and Torres, 1993; Stevens et al., 1996; Grant et al., 2000; Van Boven et al., 2000). The tactile perception of blind and sighted subjects has been compared on a variety of active (finger scanning) and passive (finger stationary) paradigms, but accurate assessment of tactile acuity is technically challenging. Active paradigms may be confounded by differences in the motor strategies used by blind and sighted subjects. Passive paradigms have often made use of tasks, such as two-point discrimination, that suffer from unintended nonspatial cues (for discussion, see Craig and Johnson, 2000). Furthermore, in all tests of passive tactile acuity of blind persons to date, the investigators have pressed the stimulus surface onto the subjects' skin by hand. Unfortunately, manual application may lead to unintended variability in such stimulus parameters as contact onset velocity, force, stability, and duration.

\footnotetext{
Received Nov. 26, 2002; revised Jan. 28, 2003; accepted Jan. 29, 2003.

This work was supported by National Eye Institute Grant 1 R15 EY13649-01 and a student fellowship from the Fight for Sight research division of Prevent Blindness America. We thank T. Chadha and B. Stavisky for assistance with experiments, D. Simons and K. Miller for suggestions on this manuscript, and D. Gillespie for invaluable consultation throughout the project.

Correspondence should be addressed to Daniel Goldreich, Occupational Therapy Department, Rangos School of Health Sciences, Duquesne University, 600 Forbes Avenue, Pittsburgh, PA 15282-0020. E-mail: goldreich@duq.edu. Copyright $\odot 2003$ Society for Neuroscience $\quad 0270-6474 / 03 / 233439-07 \$ 15.00 / 0$
}

Our goals were to determine whether blind people have enhanced passive tactile acuity and, if so, to gain insight into the responsible neural mechanism. To eliminate stimulus variability and nonspatial cues, we designed a fully automated testing system to compare the passive tactile acuity of blind and sighted subjects on a grating orientation task (Johnson and Phillips, 1981; Craig, 1999). Acuity was significantly superior in blind subjects, independently of their degree of childhood vision, light perception level, or Braille reading. The results argue against unimodal somatosensory plasticity as the cause of acuity enhancement but leave open the possibility that crossmodal plasticity plays a role.

\section{Materials and Methods \\ Subjects}

Ninety subjects were tested, 47 sighted (20.6-71.6 years old; median age of 44.2 years; 24 women and 23 men) and 43 blind (19.7-71.0 years old; median age of 48.5 years; 22 women and $21 \mathrm{men}$ ). With the exception of two blind subjects with well managed epilepsy, no subjects had CNS or PNS disorders. Blindness of cerebral origin was an exclusion criterion, as was diabetes (because of associated peripheral neuropathy). Dyslexia was an exclusion criterion for sighted subjects, because it is associated with an elevation of tactile thresholds on the grating orientation task (Grant et al., 1999). All subjects signed an informed consent form (read aloud to blind subjects) before testing; all experimental procedures were approved by the Institutional Review Board of Duquesne University for the protection of human subjects.

The blind group was heterogeneous with respect to degree of Braille reading, light perception level, and childhood (birth to 12 years old) visual experience (for numbers of subjects in subgroups, see Fig. 5). Subjects with residual light perception were unable to read print, even with magnification devices. Subjects with normal childhood vision were fully sighted throughout childhood. Those with low childhood vision were visually impaired at birth but able to read print with magnification throughout childhood. Those subjects classified with residual childhood vision were either born with residual light perception $(n=9)$ or progressed to residual light perception or less during childhood $(n=4)$. Finally, subjects with no childhood vision were born without light perception or lost all light perception within the first few months of life. We did not categorize subjects according to their age-at-onset of blindness, because this was indeterminate in a large proportion of subjects, who lost vision progressively over the course of many years.

Eleven blind subjects had participated on an early version of the task. 
Concerned that small finger movements might have facilitated tactile perception in these early experiments, we modified the task to better prevent finger movements (see below) and recalled these subjects to the laboratory for retesting 1 year later. Performance did not differ significantly between the two testing conditions (within-subject mean difference in average thresholds, $0.03 \mathrm{~mm}$ ), and test-retest thresholds were well correlated ( $r=0.84)$. Nevertheless, we include only the latter, bettercontrolled data in this report.

\section{Psychophysical procedure}

We assessed the ability of subjects to discern the orientation of grooved surfaces pressed into the stationary fingertip. Unlike two-point discrimination, which has been criticized for the presence of nonspatial cues (Johnson and Phillips, 1981), the grating orientation task requires the subject to distinguish between two stimuli that are identical with respect to contact area, force, and pressure; the stimuli differ only with respect to spatial orientation (see below). Although anisotropy has been reported in some studies (Essock et al., 1992; Wheat and Goodwin, 2000), the grating orientation task is generally considered a rigorous test of passive tactile spatial acuity (Johnson and Phillips, 1981; Craig, 1999).

The stimulus surfaces were square-wave gratings (equal groove and ridge widths) milled in the ends of $12.7 \mathrm{~mm}$ ( 0.5 inch) diameter Delrin plastic cylindrical rods (Small Parts, Miami Lakes, FL). Groove depth was sufficient to prevent the skin from reaching the bottom of a groove. Twenty pairs of gratings were used, with groove widths ranging from 0.25 to $3.10 \mathrm{~mm}$ in $0.15 \mathrm{~mm}$ increments. The gratings in a pair had identical groove widths, but one contacted the finger with the grooves parallel and the other with the grooves transverse to the axis of the finger. A Macintosh G3 computer (Apple Computers, Cupertino, CA) running Labview 5.0 (National Instruments, Austin, TX) controlled a stepper motor and linear actuator (Danaher Precision Systems, Salem, NH) to apply the gratings to the distal pad of the index finger with $4 \mathrm{~cm} / \mathrm{sec}$ onset velocity, $1 \mathrm{sec}$ duration, and 10 or 50 gm contact force (Fig. 1).

All subjects were tested on a single index finger. For sighted subjects and non-Braille readers, this was the index finger of the dominant hand, as assessed by a handedness survey (modified from Oldfield, 1971); for Braille readers, it was the index finger of the hand preferred for Braille reading. On the basis of these criteria, 30 blind subjects were tested on the right and 13 on the left index finger; 45 sighted subjects were tested on the right and two on the left index finger. The tested index finger was identified as the preferred reading finger by all Braille readers except three: two multifinger readers who expressed no finger preference and one inexperienced Braille reader who preferred to read with the little finger. Subjects held the index finger relaxed, gently flexed, and angled slightly forward and downward, the same posture used in Braille reading. Because scanning motion of the finger greatly facilitates the detection of a grooved surface, we prevented lateral and forward finger movements with plastic barriers. Downward, backward, or upward movements, detected by a sensor on the fingernail (micro switch FS; Honeywell, Freeport, IL), resulted in an error tone and the disqualification of the trial.

We used a two-interval two-alternative forced-choice up-down transformed response procedure (Levitt, 1970; Gescheider, 1997) to determine the width of the grating whose orientation the subject could distinguish with $71 \%$ probability (the $71 \%$ threshold) (Fig. 2). In each trial, the two gratings of a pair (see above) were presented sequentially (1 sec contact duration, 2 sec intercontact interval), with the presentation order chosen randomly [either grooves parallel to finger followed by transverse $(A)$ or transverse followed by parallel $(B)]$. The subject indicated the perceived stimulus order $(A$ or $B$ ) by pressing a response key with the nontested hand. Immediate auditory feedback was provided. Grating groove width began at $1.45 \mathrm{~mm}$ (an intermediate value), was stepped down after two sequential correct responses, and stepped up after a single incorrect response. This procedure produces runs of increasing and decreasing groove width whose endpoints (reversal points) bracket the $71 \%$ threshold (Levitt, 1970). Step size was $0.30 \mathrm{~mm}$ for the first three runs (to converge rapidly onto the subject's approximate threshold) and $0.15 \mathrm{~mm}$ thereafter (to determine the threshold with precision). The experimental block was terminated on completion of run 14 , and the groove widths at endpoints 4 through 14 averaged to find the $71 \%$ threshold. Threshold is unaffected by the choice of starting groove width, because the first three endpoints are not entered into the calculation. If a subject answered incorrectly on the largest groove width available $(3.1 \mathrm{~mm})$ or correctly twice in succession on the smallest groove width $(0.25 \mathrm{~mm})$, the block was terminated, the final run was assigned an end value equal to the corresponding groove width, and threshold was determined by averaging the endpoints of run 4 through the terminal run. Each subject completed 10 blocks, five with $50 \mathrm{gm}$ and five with $10 \mathrm{gm}$ contact force, in alternating order. The average number of trials per block was 43 . The average time per block was $8 \mathrm{~min}$. The entire testing session (including rest periods) lasted $\sim 2 \mathrm{hr}$.

\section{Data analysis}

We used numerical Bayesian parameter estimation to generate statistical inferences regarding within-model parameter values (effects of practice, contact force, age, gender, and blindness). Bayesian parameter estimation (Gelman et al., 1995; Sivia, 1996; Lee, 1997) produces a posterior probability density function (posterior PDF) for parameter $X$ given data $D: P(X \mid D)$. The area under the posterior PDF between any two points on the $x$-axis is the probability that the value of the parameter lies within the interval bounded by those points. The shortest $95 \%$ (or $99 \%$ ) confidence interval (CI) is the shortest $x$-axis interval for which this area is $95 \%$ (or 99\%). A parameter was considered significantly different from zero when the $95 \%$ CI did not cross zero and highly significantly different from zero when the $99 \%$ CI did not cross zero. In such cases, the mode of the posterior PDF was given as the best estimate of the value of the parameter (effect size) and the corresponding confidence interval (95\% or 99\%) reported.

We used numerical Bayesian model comparison to choose from

A

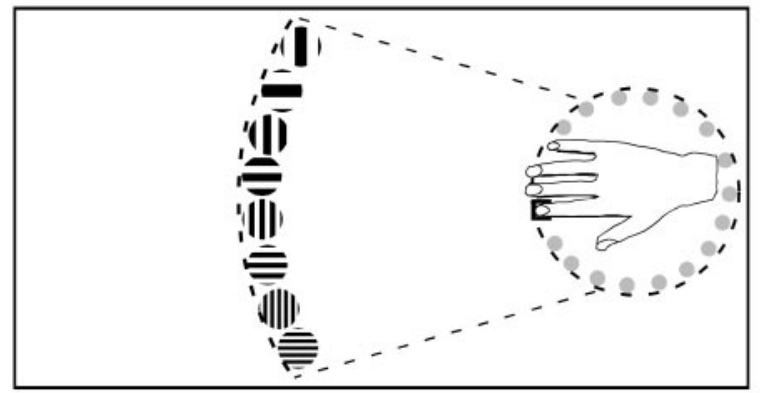

Upper Table (Top View)

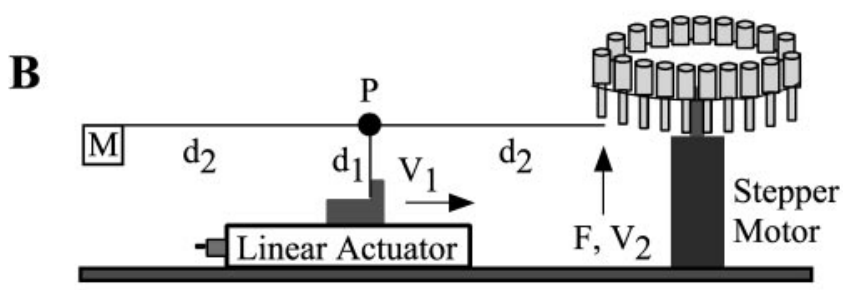

Lower Table (Side View)

Figure 1. Testing system schematic. A, Top view. The hand rested palm-down on an upper table, with the index finger positioned over a tunnel (not shown) through which the stimulus surfaces rose. Plastic barriers surrounding the fingertip prevented lateral and forward finger movements. Upward, downward, and backward movements were detected by a force sensor on the fingernail (not shown) and were announced by an error tone. A rotatable disk (dashed circle) just below the table housed the stimulus surfaces (partial expanded view on left). $B$, Side view of the lower table, concealed during testing. Rotation of the stepper motor positioned the appropriate stimulus surface under the finger, after which the linear actuator moved with velocity $V_{1}$, freeing the rod supporting mass $M$ to pivot around point $P$. The stimulus surface contacted the finger with force $F=M g$ and velocity $V_{2}=\left(V_{1}\right)\left(d_{2} / d_{1}\right)$. After a 1 sec contact period, the actuator retracted to return the rod and stimulus surface to their resting positions. $M=10$ or $50 \mathrm{gm} ; V_{2}=4 \mathrm{~cm} / \mathrm{sec}$. For clarity, only 19 of the 40 stimulus surfaces are shown. 
A $\quad$ B

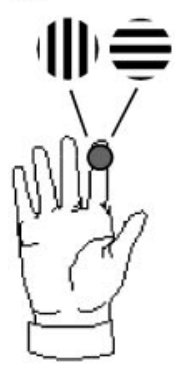

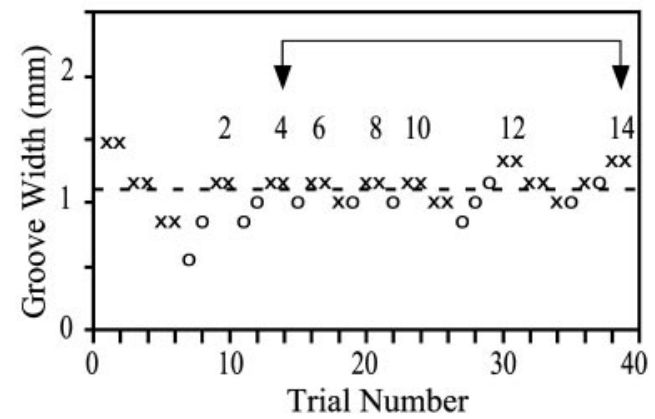

Figure 2. Experimental procedure. $A$, A single trial. Two grooved surfaces with identical groove widths but orthogonal orientations (grooves either parallel or transverse to the axis of the finger) sequentially contacted the distal pad of the index finger ( 1 sec contact duration, $2 \mathrm{sec}$ intercontact interval) in random order. The subject indicated the perceived order by pressing one of two response keys with the other hand. $B, A$ single block. Groove width was decreased after two correct $(X)$ responses and increased after a single incorrect $(O)$ response. The block was terminated on the 14th reversal of direction in groove width adjustment (top numbers). The average of groove widths at reversal points 4 through 14 (bracketed region) was the subject's threshold (dotted line; $1.10 \mathrm{~mm}$ in this case) (see Materials and Methods). The block shown was performed by a 47 -year-old blind woman at $50 \mathrm{gm}$ contact force. Each subject completed 10 such blocks, five at $50 \mathrm{gm}$ contact force alternating with five at $10 \mathrm{gm}$.

among competing statistical models (to determine whether childhood visual experience, light perception level, Braille reading, or any combinations of these factors influenced tactile acuity among the blind subjects). Bayesian model comparison (Gelman et al., 1995; Sivia, 1996; Lee, 1997 ) is uniquely suited for comparisons of simpler models (those containing fewer free parameters) to more complex ones. It produces an odds for model $\mathrm{M}_{2}$ relative to model $\mathrm{M}_{1}: P\left(\mathrm{M}_{2} \mid D\right) / P\left(\mathrm{M}_{1} \mid D\right)$. Model $\mathrm{M}_{2}$ was considered to be significantly favored over $\mathrm{M}_{1}$ when the odds were $>10$-to- 1 in its favor.

In the expressions that follow, subscript $k$ indexes subject group $(k=B$ for blind; $k=S$ for sighted), and $i$ indexes subjects. $N$ refers to group numbers $\left(N=90 ; N_{B}=43 ; N_{S}=47\right)$. For simplicity of presentation, all PDFs are expressed in un-normalized form (hence, the constant of proportionality, $\alpha$, rather than an equals sign). The total area under each PDF was subsequently normalized to 1 .

Repeated testing. We fit each subject's five thresholds measured at each contact force by linear regression against testing block number, modeled the best-fit slopes $\left(\left\{m_{i}\right\}\right)$ within each subject group as normally distributed with unknown mean $(\mu)$ and $\operatorname{SD}(\sigma)$ specified by uniform prior probability densities, and calculated the posterior PDF for the mean:

$$
P\left(\mu_{k} \mid\left\{m_{k, i}\right\}\right) \propto \int_{\sigma} \sigma^{-N_{k}} \exp \left[-\frac{1}{2 \sigma^{2}} \sum_{i=1}^{N_{k}}\left(\mu_{k}-m_{k, i}\right)^{2}\right] d \sigma
$$

Contact force. We modeled the difference scores $\left(\left\{d_{i}\right\}\right)$ between the individual subjects' 50 and $10 \mathrm{gm}$ average thresholds within each group as normally distributed with unknown mean $(\mu)$ and SD $(\sigma)$ specified by uniform prior probability densities and calculated the posterior PDF for the mean:

$$
P\left(\mu_{k} \mid\left\{d_{k, i}\right\}\right) \propto \int_{\sigma} \sigma^{-N_{k}} \exp \left[-\frac{1}{2 \sigma^{2}} \sum_{i=1}^{N_{k}}\left(\mu_{k}-d_{k, i}\right)^{2}\right] d \sigma
$$

We compared the effect of contact force in the blind group with its effect in the sighted group by using cross-correlation to generate a PDF for the difference of effects:

$$
P\left(\mu_{S}-\mu_{B}=\Delta \mid\left\{d_{i}\right\}\right)=\int_{\mu_{B}} P\left(\mu_{S}=\mu_{B}+\Delta \mid\left\{d_{S, i}\right\}\right) P\left(\mu_{B} \mid\left\{d_{B, i}\right\}\right) d \mu_{B}
$$

Visual status, age, and gender. (1) To determine whether the effects of age and gender in the blind group were equivalent to the effects of those variables in the sighted group, we modeled subjects' thresholds $\left(\left\{t_{i}\right\}\right)$ within each group as normally distributed with $\mathrm{SD}(\sigma)$ around expected values $\left(\left\{\left\langle t_{i}\right\rangle\right\}\right)$ formed by a linear combination of subject age $(a)$ and gender $(g)$ :

$$
\left\langle t_{k, i}\right\rangle=\left(C_{1 k}\right)\left(a_{k, i}\right)+\left(C_{2 k}\right)\left(g_{k, i}\right)+C_{3 k}
$$

where parameters $C_{1}, C_{2}, C_{3}$, and $\sigma$ were specified by uniform prior probability densities. We calculated posterior PDFs for parameters $C_{1}$ and $C_{2}$ of each group and then used cross-correlation to generate PDFs for the differences between the blind and corresponding sighted parameters:

$$
\begin{array}{r}
P\left(C_{1 k} \mid\left\{t_{k, i}\right\}\right) \propto \int_{C_{2 k}} \int_{C_{3 k}} \int_{\sigma} \sigma^{-N_{k}} \exp \left[-\frac{1}{2 \sigma^{2}} \sum_{i=1}^{N_{k}}\left(\left\langle t_{k, i}\right\rangle-t_{k, i}\right)^{2}\right] d C_{2 k} d C_{3 k} d \sigma \\
P\left(C_{1 S}-C_{1 B}=\Delta \mid\left\{t_{i}\right\}\right)=\int_{C_{1 B}} P\left(C_{1 S}=C_{1 B}\right. \\
\left.+\Delta \mid\left\{t_{S, i}\right\}\right) P\left(C_{1 B} \mid\left\{t_{B, i}\right\}\right) d C_{1 B}
\end{array}
$$

and correspondingly for $C_{2 S}$ and $C_{2 B}$.

(2) To assess the effects of visual status ( $v$, blind or sighted), age $(a)$, and gender $(g)$, we used a main-effects analysis of covariance (ANCOVA) [chosen because (1) above revealed that age affected the two subject groups equivalently, as did gender]. Subjects' thresholds $\left(\left\{t_{i}\right\}\right)$ were modeled as normally distributed with $\mathrm{SD}(\sigma)$ around expected values $\left(\left\{<t_{i}>\right\}\right)$ formed by a linear combination of these variables:

$$
\left\langle t_{i}\right\rangle=\left(C_{1}\right)\left(V_{i}\right)+\left(C_{2}\right)\left(a_{i}\right)+\left(C_{3}\right)\left(g_{i}\right)+C_{4}
$$

where parameters $C_{1}$ through $C_{4}$ and $\sigma$ were specified by uniform prior probability densities. The posterior PDF was calculated for parameter $C_{1}$ as follows:

$$
P\left(C_{1} \mid\left\{t_{i}\right\}\right) \propto \int_{C_{2}} \int_{C_{3}} \int_{C_{4}} \int_{\sigma} \sigma^{-N} \exp \left[-\frac{1}{2 \sigma^{2}} \sum_{i=1}^{N}\left(\left\langle t_{i}\right\rangle-t_{i}\right)^{2}\right] d C_{2} d C_{3} d C_{4} d \sigma
$$

and correspondingly for $C_{2}$ and $C_{3}$.

Childhood vision, light perception, and Braille reading. We used Bayesian model comparison to determine whether the data were best explained by the factors age, gender, and vision (sighted or blind) only ( $\operatorname{model} \mathrm{M}_{1}$ ) or by models $\left(\mathrm{M}_{2}\right)$ that, in addition to age, gender, and vision, also considered blind subjects' childhood visual experience, light perception level, and Braille reading. We calculated odds, $P\left(\mathrm{M}_{2} \mid D\right) / P\left(\mathrm{M}_{1} \mid D\right)$, for seven models $\mathrm{M}_{2}$ consisting of all possible combinations of one, two, or three of the factors childhood vision, light perception, and Braille reading, and for an additional 31 models $\mathrm{M}_{2}$ consisting of all possible combinations of childhood vision, light perception, age-at-learning Braille, years reading Braille, and daily Braille reading hours.

Model $\mathrm{M}_{1}$ was represented as

$$
\left\langle t_{i}\right\rangle=\left(C_{1}\right)(a)+\left(C_{2}\right)(g)+C_{3}-\left(C_{4}\right)(b l),
$$

whereas models $\mathrm{M}_{2}$ were represented as the seven possible variations of

$$
\begin{aligned}
\left\langle t_{i}\right\rangle=\left(C_{1}\right)(a)+\left(C_{2}\right)(g)+C_{3}+(b l)\left[-C_{4}+\left(C_{5}\right)(c v)\right. & +\left(C_{6}\right)(l p) \\
& \left.-\left(C_{7}\right)(b r)\right],
\end{aligned}
$$

and the 31 possible variations of

$$
\begin{aligned}
\left\langle t_{i}\right\rangle=\left(C_{1}\right)(a)+\left(C_{2}\right)(g)+C_{3}+ & (b l)\left[-C_{4}+\left(C_{5}\right)(c v)+\left(C_{6}\right)(l p)\right. \\
& \left.+\left(C_{7}\right)\left(b r_{a}\right)-\left(C_{8}\right)\left(b r_{y}\right)-\left(C_{9}\right)\left(b r_{h}\right)\right]
\end{aligned}
$$

where variables $a$ and $g$ represented age and gender; $b l$ was 1 (blind) or 0 (sighted); $c v, l p$, and $b r$ represented childhood vision [0(none), 1(resid- 
ual), 2(low), or 3(normal)], light perception level [0(none), 1(residual)], and Braille [0(nonreader), 1(reader)]; and $b r_{a}, b r_{y}$ and $b r_{h}$ represented age-at-learning Braille, years reading Braille, and daily Braille reading hours, respectively. For clarity, we omitted the subscript $i$ from each of the variables in the above three equations.

The different model $\mathrm{M}_{2}$ variations were formed by eliminating parameters within the brackets in the above expressions to produce models with all possible subsets of parameters $C_{5}$ and greater. All variables within brackets were normalized to range from 0 to 1 , and parameters $C_{4}$ and above were specified by one-sided uniform prior probability densities over the range $0-0.5 \mathrm{~mm}$. One-sided prior probability densities were used (in this multifactorial analysis only) to maximize the probability of detecting effects in the expected direction (indicated by the sign preceding each parameter). Models $\mathrm{M}_{1}$ and $\mathrm{M}_{2}$ were assigned equal prior probabilities. Thus, the odds $P\left(\mathrm{M}_{2} \mid D\right) / P\left(\mathrm{M}_{1} \mid D\right)$ were equal to the marginal likelihood ratios $P\left(D \mid \mathrm{M}_{2}\right) / P\left(D \mid \mathrm{M}_{1}\right)$.

\section{Results}

We designed an automated system to stimulate the index fingertips of blind and sighted subjects with surfaces containing parallel grooves (Fig. 1). In each trial, two grooved surfaces with identical groove widths but orthogonal orientations sequentially contacted the distal pad of the index finger, and the subject indicated the perceived stimulus order. An adaptive method was used in which the system applied narrower (more difficult) grooves when the subject answered correctly and wider (easier) ones when the subject answered incorrectly to determine the width of the grooves whose orientation the subject could reliably perceive (Fig. 2). This reliably perceptible groove width, the subject's tactile threshold, is inversely related to tactile spatial acuity. To investigate the influence of surface-to-skin contact force on tactile perception, we used forces of 50 and $10 \mathrm{gm}$, and, to determine whether acuity improved with short-term practice, we tested each subject five times at each contact force.

Thresholds in the blind and sighted subject groups were stable with repeated testing at each contact force, with no significant improvements observed (Fig. $3 A$ ). The average within-subject SE across 50 gm testing blocks was $0.15 \mathrm{~mm}$ (sighted) and $0.15 \mathrm{~mm}$ (blind); on $10 \mathrm{gm}$, it was $0.18 \mathrm{~mm}$ (sighted) and $0.17 \mathrm{~mm}$ (blind). Because performance was stable, we averaged each subject's five $50 \mathrm{gm}$ and five $10 \mathrm{gm}$ thresholds to investigate the effects of contact force (Fig. 3B). Contact force affected blind and sighted subjects equivalently. Within each group, individual subjects' average 10 and $50 \mathrm{gm}$ thresholds were positively correlated $(r=$ 0.60 for sighted; $r=0.65$ for blind), but the 50 gm thresholds were significantly lower. In fact, subjects could detect grooves that were nearly $0.5 \mathrm{~mm}$ narrower when the stimulus surface was pressed more firmly into the skin (sighted: effect size, $0.44 \mathrm{~mm}$; 99\% CI, 0.28-0.61 mm; blind: effect size, $0.49 \mathrm{~mm}$; 99\% CI, $0.33-0.65 \mathrm{~mm})$.

To compare blind with sighted subjects, we averaged each subject's scores from all 10 testing blocks to obtain an indicator of overall performance (average tactile threshold) (Fig. 4A). An ANCOVA (Fig. $4 B$ ) revealed that blind subjects significantly outperformed their sighted peers (effect size, $0.33 \mathrm{~mm}$ lower thresholds for blind; $99 \% \mathrm{CI}, 0.14-0.53 \mathrm{~mm})$. In addition, performance declined significantly with age (effect size, $0.014 \mathrm{~mm}$ average threshold increase per year; 99\% CI, 0.007-0.022 mm/ year), and women outperformed men (effect size, $0.18 \mathrm{~mm} ; 95 \%$ CI, $0.03-0.34 \mathrm{~mm}$ ) (Fig. $4 \mathrm{~B}$ ). Because the age-related decline in acuity was equivalent for blind and sighted groups, the difference between subject groups could be expressed in age-equivalent terms: the average blind subject had the acuity of an average sighted subject of the same gender but 23 years younger (Fig. 4C).
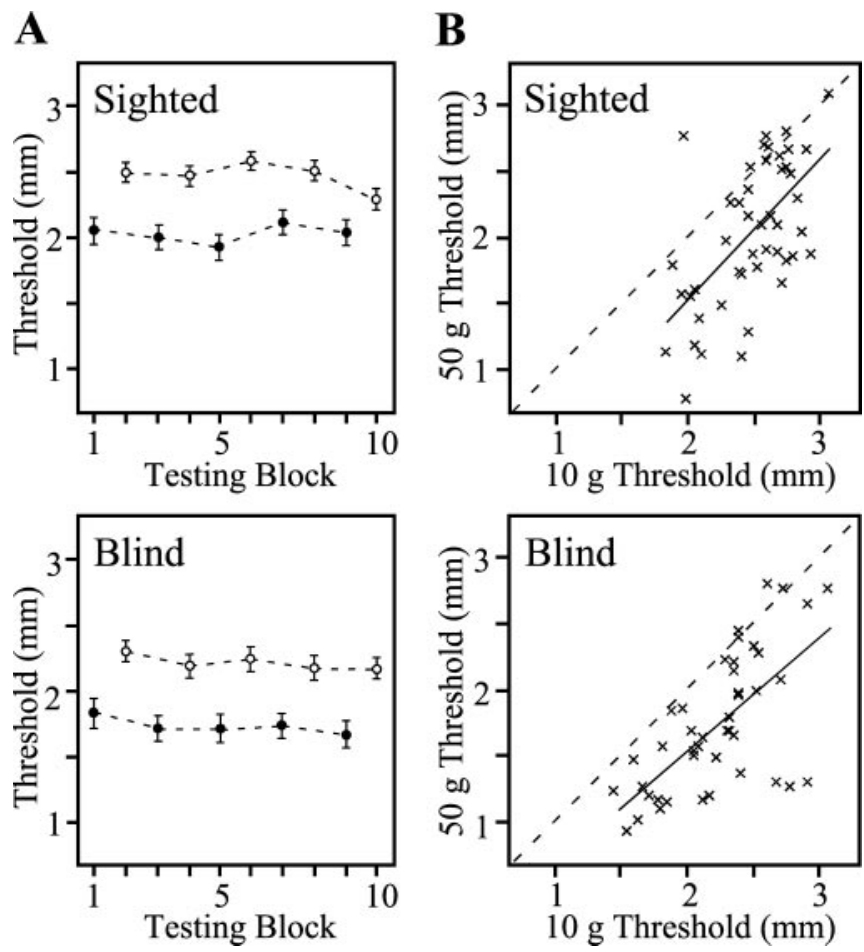

Figure 3. Effects of repeated testing and contact force. $A$, Group means \pm SEs of sighted and blind subjects on the five $50 \mathrm{gm}$ (filled symbols) and five $10 \mathrm{gm}$ (open symbols) testing blocks. $B$, Scatter plot showing effect of contact force. Each point represents the average of a subject's five $10 \mathrm{gm}$ thresholds plotted against the average of the subject's five $50 \mathrm{gm}$ thresholds. Dashed lines, $x=y$; solid lines, best linear fits.

Blind subjects significantly outperformed sighted subjects at both the $10 \mathrm{gm}$ (effect size, $0.30 \mathrm{~mm}$; 99\% CI, 0.11-0.47 $\mathrm{mm}$ ) and 50 gm (effect size, $0.37 \mathrm{~mm}$; 99\% CI, 0.10-0.64 mm) contact forces.

We next used ANCOVAs to compare the average thresholds of sighted subjects with those of eight subgroups of blind subjects (Fig. 5A). Each blind subgroup had significantly greater tactile acuity than the sighted group: subjects with no childhood vision (effect size, $0.46 \mathrm{~mm}$; 99\% CI, $0.14-0.78 \mathrm{~mm}$ ), residual childhood vision (effect size, $0.22 \mathrm{~mm}$; 95\% CI 0.01-0.44 mm), low childhood vision (effect size, $0.52 \mathrm{~mm}$; 99\% CI, 0.18-0.85 mm), normal childhood vision (effect size, $0.27 \mathrm{~mm}$; 95\% CI, $0.04-$ $0.49 \mathrm{~mm}$ ), no light perception (effect size, $0.40 \mathrm{~mm} ; 99 \% \mathrm{CI}$, $0.16-0.62 \mathrm{~mm}$ ), residual light perception (effect size, $0.24 \mathrm{~mm}$; 95\% CI, 0.04-0.44 mm), Braille nonreaders (effect size, 0.35 $\mathrm{mm}$; 99\% CI, 0.01-0.69 mm), Braille readers (effect size, 0.34 $\mathrm{mm} ; 99 \% \mathrm{CI}, 0.12-0.54 \mathrm{~mm}$ ). Thus, tactile acuity enhancement was not restricted to subjects who were blind as children, to those who completely lacked light perception, or to Braille readers. In fact, even the five blind subjects in our sample who had normal childhood vision, residual light perception, and read no Braille significantly outperformed the sighted subjects (effect size, 0.39 $\mathrm{mm}$; 95\% CI, 0.08-0.70 mm) (data not shown).

In view of these results, we asked the following question: to what degree, if any, do childhood visual experience, light perception level, and Braille reading influence the tactile acuity of blind subjects? To answer this question, we used a multivariate analysis to assess the influence of each of these three factors, both individually and in all possible factorial combinations. Interestingly, none of the factors (Fig. 5A) significantly affected blind subjects' tactile thresholds (odds for all seven models, $<1.5$; see Materials and Methods). To further explore whether Braille reading expe- 
A

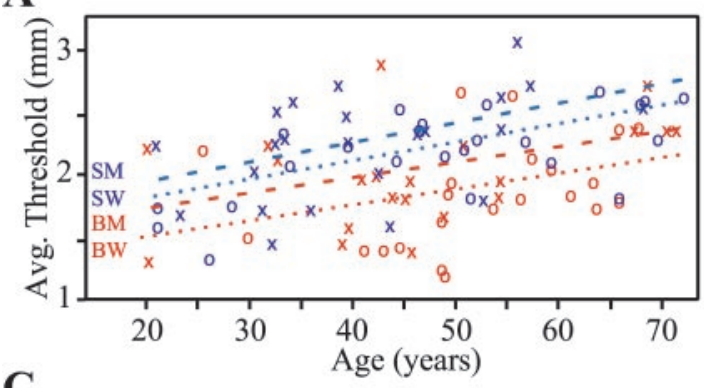

C

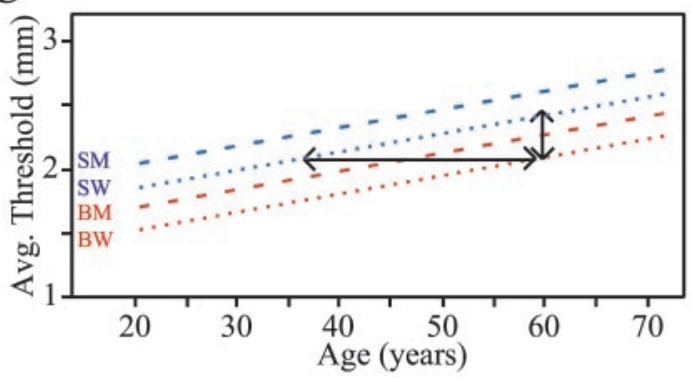

B
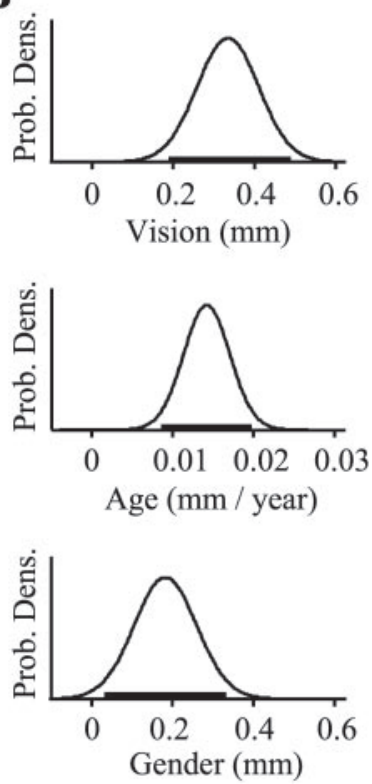

Figure 4. Effects of blindness, age, and gender. A, Each subject's average threshold (average of all 10 testing blocks) plotted against age. Blind, Red; sighted, blue; women, $\bigcirc$; men, $\times$. Best-fit linear regression lines are shown for sighted men (SM), sighted women (SW), blind men (BM), and blind women (BW). B, Results of ANCOVA showing posterior PDFs for the effects of vision, age, and gender. Horizontal bars indicate $95 \%$ confidence intervals. C, Best estimates for the effects of vision, age, and gender, representing the modes of the PDFs from $B$. The $0.33 \mathrm{~mm}$ difference between blind and sighted subjects of the same gender (vertical arrows) is equivalent to an age difference of 23 years (horizontal arrows).

rience might influence tactile acuity, we categorized Braille readers according to their age-at-learning Braille, years reading Braille, and daily Braille reading hours (Fig. 5B). Again, none of these Braille factors correlated with tactile acuity (odds for all 31 models, $<1.5$; see Materials and Methods).

\section{Discussion}

We showed that passive tactile acuity is significantly superior in blind subjects and is enhanced independently of degree of childhood vision, light perception level, or Braille reading. Furthermore, acuity depends strongly on contact force, declines with subject age, and is better in women than in men. Despite large intragroup variability, the difference between blind and sighted subjects was highly significant: the average blind subject had the acuity of an average sighted subject of the same gender but 23 years younger.

\section{Comparison with previous psychophysical studies}

Whether blind persons have enhanced tactile acuity has long been controversial. Two previous grating orientation studies reached opposing conclusions. Van Boven et al. (2000) tested 15 early blind Braille readers and 15 sighted subjects, manually applying the gratings to the index and middle fingers of both hands, and concluded that the blind subjects were superior to the sighted on all four fingers. Grant et al. (2000) tested 15 early blind Braille readers, 9 late blind Braille readers, and 25 sighted subjects, manually applying the gratings to the index fingers of both hands, and concluded that the three groups did not differ on either finger. Our finding of superior acuity in blind subjects confirms that of Van Boven et al. (2000); it is also consistent with Stevens et al. (1996), who found superior performance in blind subjects on several nongrating measures of passive tactile acuity.

We observed a highly significant effect of contact force on tactile acuity among both blind and sighted subjects (Fig. 3).
Similarly, Johnson and Phillips (1981) report improvement in performance on grating orientation discrimination as a function of increasing skin indentation. The slowly adapting type I (SA-I) cutaneous receptor population conveys the highresolution spatial information required for grating perception (Phillips and Johnson, 1981). The firing rates of SA-I afferents rise nearly linearly with increasing skin indentation (Vega-Bermudez and Johnson, 1999), presumably facilitating the discrimination of grating orientation at higher contact forces. The $50 \mathrm{gm}$ thresholds of the blind and sighted subjects in the present study are somewhat higher than those reported by Van Boven et al. (2000); this difference is probably attributable to the use of larger contact forces in that study.

Whereas the superior tactile acuity of blind persons likely has a central explanation (see below), the effects of gender and age may be peripheral in origin. The heightened tactile acuity of women (Fig. 4), also found (among blind subjects) by Van Boven et al. (2000), may be attributable to greater skin compliance (Woodward, 1993). The parallel age-related decline in tactile acuity among blind and sighted subjects (Fig. 4), also found by Stevens et al. (1996), may be attributable to peripheral and/or central neuronal loss with age.

\section{Evidence against a childhood critical period for tactile acuity enhancement}

Our data indicate that early blindness is not necessary for enhanced tactile acuity. Blind subjects who had normal vision throughout childhood significantly outperformed sighted subjects (Fig. 5A). Furthermore, degree of childhood vision neither correlated significantly with tactile acuity among blind subjects nor showed a monotonic trend in this regard (Fig. 5A). Thus, our data argue against the importance of childhood visual deprivation in tactile acuity enhancement.

\section{Evidence that loss of sight itself drives tactile acuity enhancement}

Blindness is characterized by both loss of sight and increased reliance on the nonvisual senses. Our results suggest that loss of sight in itself leads to acuity enhancement, whereas the accompanying tactile experience exerts little influence on tactile acuity. This conclusion follows from the observation that Braille reading experience did not correlate with tactile acuity among our blind subjects. Braille readers, although tested on the preferred reading finger, performed equivalently to blind nonreaders (Fig. 5A), and no significant effects were observed of the age at which Braille was learned, years reading Braille, or daily Braille reading time (Fig. $5 B$ ). The lack of effect of Braille reading is all the more striking considering that Braille reading, like grating orientation discrimination, may rely in large part on SA-I receptor activity (Phillips et al., 1990). In light of the statistically insignificant effect of Braille reading, it would seem unlikely that the enhanced tactile acuity of blind persons results primarily from enriched tactile 
A

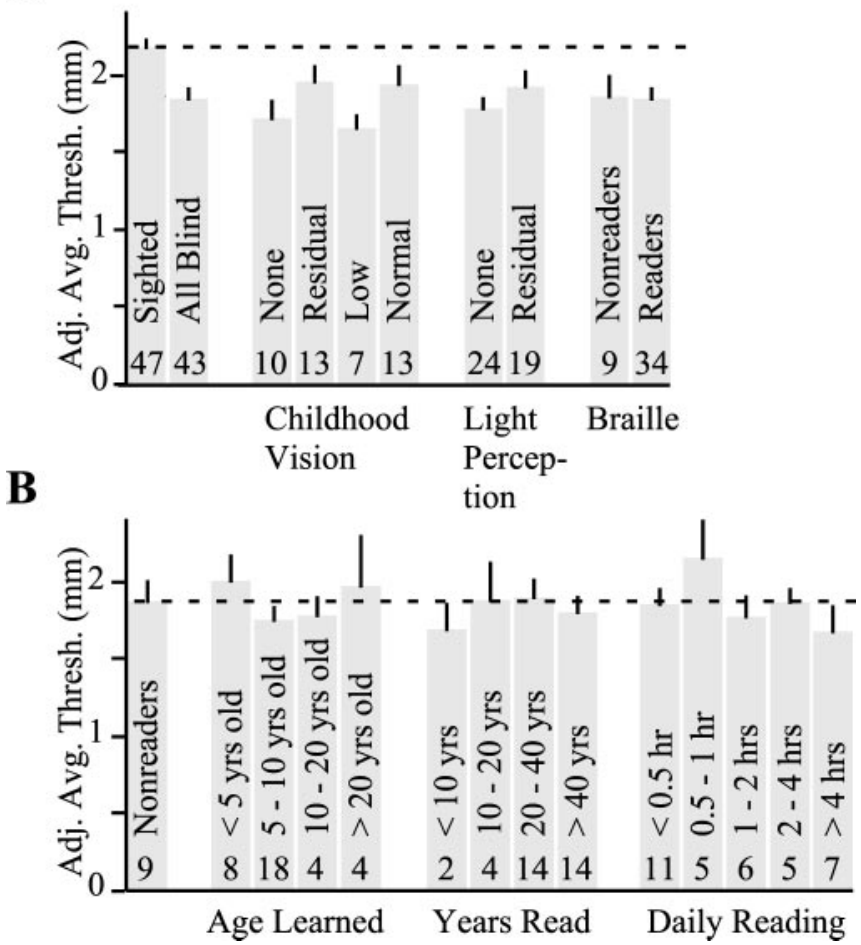

Figure 5. Effects of childhood visual experience, light perception level, and Braille reading. For display purposes only, each subject's average threshold was age adjusted (at $0.014 \mathrm{~mm}$ / year) to 45 years old, and thresholds of men were gender adjusted (by subtracting $0.018 \mathrm{~mm}$ ) to equivalent female values. $A$, Adjusted average thresholds of sighted (left; dashed line) and different subgroups of blind subjects. $B$, Adjusted average thresholds of blind nonreaders (left; dashed line) and different subgroups of Braille readers. Histogram bars indicate group means, error bars indicate SEs, and numbers indicate group sizes.

experience. We cautiously favor a more parsimonious interpretation of our data: that severe visual deprivation (to the level of residual light perception or less) itself results in tactile acuity gains.

The conclusion that tactile acuity is enhanced by the loss of sight per se receives support from a study that compared the effect of Braille training with that of long-term blindfolding in sighted subjects (Kauffman et al., 2002). Using a two-by-two factorial design, the authors found that passive tactile acuity on the right index finger was unaffected by $5 \mathrm{~d}$ of intensive $(4 \mathrm{hr} / \mathrm{d})$ Braille training. In contrast, acuity on this finger was significantly enhanced by a $5 \mathrm{~d}$ period of blindfolding. Additional support for this conclusion comes from Van Boven et al. (2000), who found no correlation between Braille reading experience and tactile acuity on the reading finger. Furthermore, even on their nonreading fingers, the Braille readers in that study (like our nonreaders) had significantly greater acuity than sighted subjects.

Nevertheless, Van Boven et al. (2000) report that, of 13 Braille readers with a preferred reading finger, nine had greater acuity on that finger than on three other fingers tested. Although this result is consistent with the hypothesis that Braille reading improves tactile acuity on the reading finger, a plausible alternative hypothesis is that Braille readers prefer to read with the finger that has greatest (preexisting) acuity. In sighted subjects, acuity varies unsystematically by several tenths of a millimeter between homologous fingers on the two hands, and, although acuity decreases on average from index to ring finger, substantial non-monotonic acuity fluctuations among the fingers of an individual hand are not uncommon (Vega-Bermudez and Johnson, 2001). The source of inter-finger acuity variability is unknown, but variation in peripheral receptor density is plausible. In any event, because greater tactile acuity apparently enables more rapid Braille reading (Stevens et al., 1996), Braille readers might naturally prefer to read with the finger that has greatest acuity. To distinguish between these hypotheses, longitudinal studies should be done to track blind individuals' tactile acuity on reading and nonreading fingers during Braille acquisition.

\section{Speculations on the neural mechanism of tactile acuity enhancement}

Our results suggest that unimodal somatosensory plasticity is not the primary mechanism of acuity enhancement in blindness. Braille reading causes a progressive expansion of the sensory representation of the reading finger within primary somatosensory (S-1) cortex (Pascual-Leone and Torres, 1993; Sterr et al., 1999), whereas representations of the nonreading fingers are apparently unaffected (Pascual-Leone and Torres, 1993). Given our finding that tactile acuity does not correlate with Braille reading experience, it seems unlikely that the observed representational expansions within S-1 contribute significantly to passive tactile acuity. The functional consequence of such expansions is unclear. The representation of the reading finger in motor cortex is also enlarged (Pascual-Leone et al., 1993); perhaps the somatosensory expansion reflects increased connectivity between somatosensory and motor areas that develops to coordinate the sensory and motor aspects of Braille reading. The lack of effect on tactile acuity of unimodal somatosensory plasticity is less surprising given the recent finding that the amputation of a finger does not result in heightened acuity of the intact, adjacent fingers (VegaBermudez and Johnson, 2002), despite the fact that the central representations of those fingers expand dramatically (Merzenich et al., 1984).

What, then, is the source of the tactile superiority of blind persons? Crossmodal plasticity may play a role. In blind humans and experimentally blinded animals, normally visually responsive cortical areas are activated by nonvisual stimuli (Rauschecker, 1995). Functional imaging studies have shown activation of extrastriate cortical areas in early and, to a lesser extent, late blind subjects performing tactile tasks (Cohen et al., 1999; Sadato et al., 2002). According to several studies, primary visual cortex is activated only in early blind subjects (Sadato et al., 1996, 1998, 2002; Cohen et al., 1999) (but see Büchel et al., 1998; Burton et al., 2002), yet tactile stimuli activated both striate and extrastriate cortex in sighted adults blindfolded for $5 \mathrm{~d}$ (PascualLeone and Hamilton, 2001). In several studies, the functional relevance of tactile occipital activation was confirmed through the use of disruptive transcranial magnetic stimulation (Cohen et al., 1997, 1999; Pascual-Leone and Hamilton, 2001). Crossmodal plasticity may occur when a normally subtle tactile influence within occipital visual areas (Zangaladze et al., 1999; Macaluso et al., 2000, 2002; Amedi et al., 2001) strengthens upon loss of competing visual inputs. Additional studies are needed to better characterize the dependence of crossmodal plasticity on the time course of blindness and to determine the perceptual consequences of crossmodal activation. The discovery of the neural mechanism underlying the enhanced tactile acuity of blind persons will deepen our understanding of sensory compensation and may ultimately lead to advances in rehabilitation strategies for patients with sensory loss. 


\section{References}

Amedi A, Malach R, Hendler T, Peled S, Zohary E (2001) Visuo-haptic object-related activation in the ventral visual pathway. Nat Neurosci 4:324-330

Axelrod S (1959) Effects of early blindness: performance of blind and sighted children on tactile and auditory tasks. New York: American Foundation for the Blind.

Büchel C, Price C, Frackowiak RSJ, Friston K (1998) Different activation patterns in the visual cortex of late and congenitally blind subjects. Brain 121:409-419.

Burton H, Snyder AZ, Conturo TE, Akbudak E, Ollinger JM, Raichle ME (2002) Adaptive changes in early and late blind: a fMRI study of braille reading. J Neurophysiol 87:589-607.

Cohen LG, Celnik P, Pascual-Leone A, Corwell B, Faiz L, Dambrosia J, Honda M, Sadato N, Gerloff C, Catalá MD, Hallett M (1997) Functional relevance of cross-modal plasticity in blind humans. Nature 389:180-183.

Cohen LG, Weeks RA, Sadato N, Celnik P, Ishii K, Hallett M (1999) Period of susceptibility for cross-modal plasticity in the blind. Ann Neurol 45:451-460.

Craig JC (1999) Grating orientation as a measure of tactile spatial acuity. Somatosens Mot Res 16:197-206.

Craig JC, Johnson KD (2000) The two-point threshold: not a measure of tactile spatial resolution. Curr Dir Psychol Sci 9:29-32.

Essock EA, Krebs WK, Prather JR (1992) An anisotropy of human tactile sensitivity and its relation to the visual oblique effect. Exp Brain Res 91:520-524.

Gelman A, Carlin JB, Stern HS, Rubin DB (1995) Bayesian data analysis. London: Chapman and Hall.

Gescheider GA (1997) Psychophysics: the fundamentals, Chap 7, Ed 3. Mahwah, NJ: Erlbaum.

Grant AC, Zangaladze A, Thiagarajah MC, Sathian K (1999) Tactile perception in developmental dyslexia: a psychophysical study using gratings. Neuropsychologia 37:1201-1211.

Grant AC, Thiagarajah MC, Sathian K (2000) Tactile perception in blind braille readers: a psychophysical study of acuity and hyperacuity using gratings and dot patterns. Percept Psychophys 62:301-312.

Hollins M (1989) Understanding blindness: an integrative approach, Chap 3. Hillsdale, NJ: Erlbaum.

Johnson KO, Phillips JR (1981) Tactile spatial resolution. I. Two-point discrimination, gap detection, grating resolution, and letter recognition. J Neurophysiol 46:1177-1191.

Kauffman T, Théoret H, Pascual-Leone A (2002) Braille character discrimination in blindfolded human subjects. NeuroReport 13:571-574.

Lee PM (1997) Bayesian statistics: an introduction. Ed 2. London: Arnold.

Lessard N, Paré M, Lepore F, Lassonde M (1998) Early-blind human subjects localize sound sources better than sighted subjects. Nature 395:278-280

Levitt H (1970) Transformed up-down methods in psychoacoustics. J Acoust Soc Am 49:467-477.

Macaluso E, Frith CD, Driver J (2000) Modulation of human visual cortex by crossmodal spatial attention. Science 289:1206-1208.

Macaluso E, Frith CD, Driver J (2002) Crossmodal spatial influences of touch on extrastriate visual areas take current gaze direction into account. Neuron 34:647-658.

Melzer P, Morgan VL, Pickens DR, Price RR, Wall RS, Ebner FF (2001) Cortical activation during braille reading is influenced by early visual experience in subjects with severe visual disability: a correlational fMRI study. Hum Brain Mapp 14:186-195.

Merzenich MM, Nelson RJ, Stryker MP, Cynader MS, Schoppmann A, Zook
JM (1984) Somatosensory cortical map changes following digit amputation in adult monkeys. J Comp Neurol 224:591-605.

Oldfield RC (1971) The assessment and analysis of handedness: the Edinburgh inventory. Neuropsychologia 9:97-113.

Pascual-Leone A, Hamilton R (2001) The metamodal organization of the brain. Prog Brain Res 134:427-445.

Pascual-Leone A, Torres F (1993) Plasticity of the sensorimotor cortex representation of the reading finger in braille readers. Brain 116:39-52.

Pascual-Leone A, Cammarota A, Wassermann EM, Brasil-Neto JP, Cohen LG, Hallett M (1993) Modulation of motor cortical outputs to the reading hand of braille readers. Ann Neurol 34:33-37.

Phillips JR, Johnson KO (1981) Tactile spatial resolution. II. Neural representation of bars, edges, and gratings in monkey primary afferents. J Neurophysiol 46:1192-1203

Phillips JR, Johansson RS, Johnson KO (1990) Representation of braille characters in human nerve fibers. Exp Brain Res 81:589-592.

Rauschecker JP (1995) Compensatory plasticity and sensory substitution in the cerebral cortex. Trends Neurosci 18:36-43.

Röder B, Teder-Salejarvi W, Sterr A, Rosler F, Hillyard SA, Neville HJ (1999) Improved auditory spatial tuning in blind humans. Nature 400:162-166.

Sadato N, Pascual-Leone A, Grafman J, Ibañez V, Deiber MP, Dold G, Hallett M (1996) Activation of the primary visual cortex by braille reading in blind subjects. Nature 380:526-528.

Sadato N, Pascual-Leone A, Grafman J, Deiber MP, Ibañez V, Hallett M (1998) Neural networks for braille reading by the blind. Brain 121:1213-1229.

Sadato N, Okada T, Honda M, Yoshiharu Y (2002) Critical period for crossmodal plasticity in blind humans: a functional MRI study. NeuroImage $16: 389-400$.

Sivia DS (1996) Data analysis: a Bayesian tutorial. New York: Oxford UP.

Sterr A, Muller M, Elbert T, Rockstroh B, Taub E (1999) Development of cortical reorganization in the somatosensory cortex of adult braille students. Electroencephalogr Clin Neurophysiol Suppl 49:292-298.

Stevens JC, Foulke E, Patterson MQ (1996) Tactile acuity, aging, and braille reading in long-term blindness. J Exp Psychol Appl 2:91-106.

Van Boven RW, Hamilton RH, Kauffman T, Keenan JP, Pascual-Leone A (2000) Tactile spatial resolution in blind braille readers. Neurology $54: 2230-2236$.

Vega-Bermudez F, Johnson KO (1999) SAl and RA receptive fields, response variability, and population responses mapped with a probe array. J Neurophysiol 81:2701-2710.

Vega-Bermudez F, Johnson KO (2001) Differences in spatial acuity between digits. Neurology 56:1389-1391.

Vega-Bermudez F, Johnson KO (2002) Spatial acuity after digit amputation. Brain 125:1256-1264.

Warren DH (1978) Perception by the blind. In: Handbook of perception, Vol X (Carterette EC, Friedman MP, eds), pp 65-90. New York: Academic.

Weeks R, Horwitz B, Aziz-Sultan A, Tian B, Wessinger CM, Cohen LG, Hallett M, Rauschecker JP (2000) A positron emission tomographic study of auditory localization in the congenitally blind. J Neurosci 20:2664-2672.

Wheat HE, Goodwin AW (2000) Tactile discrimination of gaps by slowly adapting afferents: effects of population parameters and anisotropy in the fingerpad. J Neurophysiol 84:1430-1444.

Woodward KL (1993) The relationship between skin compliance, age, gender, and tactile discriminative thresholds in humans. Somatosens Mot Res 10:63-67.

Zangaladze A, Epstein CM, Grafton ST, Sathian K (1999) Involvement of visual cortex in tactile discrimination of orientation. Nature 401:587-590. 